\title{
CORRIGENDUM TO: ASYMPTOTIC \\ SHAPE AND THE SPEED OF \\ PROPAGATION OF CONTINUOUS-TIME CONTINUOUS-SPACE BIRTH PROCESSES
}

V. BEZBORODOV, * Wroctaw University of Science and Technology

\begin{abstract}
We would like to correct the statement of Lemma 4.1 in $\left[\mathrm{BDK}^{+} 18\right]$.

Keywords: shape theorem; spatial birth process; growth model

2010 Mathematics Subject Classification: Primary 60K35

Secondary $60 \mathrm{~J} 80$
\end{abstract}

\section{Introduction}

Let $(S, \mathscr{B}(S))$ be a Polish (state) space. Consider a (time-homogeneous) Markov chain on $(S, \mathscr{B}(S))$ as a family of probability measures on $S^{\infty}$. Namely, on the measurable space $(\bar{\Omega}, \mathscr{F})=\left(S^{\infty}, \mathscr{B}\left(S^{\infty}\right)\right)$ consider a family of probability measures $\left\{P_{S}\right\}_{s \in S}$ such that for the coordinate mappings

$$
X_{n}: \bar{\Omega} \rightarrow S, \quad X_{n}\left(s_{1}, s_{2}, \ldots\right)=s_{n},
$$

the process $X:=\left\{X_{n}\right\}_{n \in \mathbb{Z}_{+}}$is a Markov chain such that for all $s \in S$

$$
\mathbb{P}_{s}\left\{X_{0}=s\right\}=1, \quad \mathbb{P}_{s}\left\{X_{n+m_{j}} \in A_{j}, j=1, \ldots, l \mid \mathscr{F}_{n}\right\}=\mathbb{P}_{X_{n}}\left\{X_{m_{j}} \in A_{j}, j=1, \ldots, l\right\} .
$$

Here $A_{j} \in \mathscr{B}(S), m_{j} \in \mathbb{N}, l \in \mathbb{N}, \mathscr{F}_{n}=\sigma\left\{X_{1}, \ldots, X_{n}\right\}$. The space $S$ is separable, hence there exists a transition probability kernel $Q: S \times \mathscr{B}(S) \rightarrow[0,1]$ such that

$$
Q(s, A)=\mathbb{P}_{s}\left\{X_{1} \in A\right\}, \quad s \in S, A \in \mathscr{B}(S) .
$$

Consider a transformation of the chain $X, Y_{n}=f\left(X_{n}\right)$, where $f: S \rightarrow \mathbb{R}$ is a Borel-measurable function. Lemma 4.1 in $\left[\mathrm{BDK}^{+} 18\right]$ gives sufficient conditions for $Y=\left\{Y_{n}\right\}_{n \in \mathbb{Z}_{+}}$to be a Markov chain. The original erroneous Lemma 4.1 had the following formulation.

Lemma 4.1 old. Assume that for any bounded Borel function $h: S \rightarrow S$

$$
\mathbb{E}_{s} h\left(X_{1}\right)=\mathbb{E}_{q} h\left(X_{1}\right) \text { whenever } f(s)=f(q) .
$$

Then $Y$ is a Markov chain.

Lemma 4.1 and the remark succeeding it in $\left[\mathrm{BDK}^{+} 18\right]$ should instead read as follows.

Lemma 4.1. Assume that for any bounded Borel function $h: S \rightarrow S$

$$
\mathbb{E}_{s} h\left(Y_{1}\right)=\mathbb{E}_{q} h\left(Y_{1}\right) \text { whenever } f(s)=f(q) .
$$

Then $Y$ is a Markov chain.

\footnotetext{
* Postal address: Wrocław University of Science and Technology, Faculty of Electronics, Janiszewskiego 11, Wrocław, Poland.
} 
Remark 4.1. Condition (1) is the equality of distributions of $Y_{1}$ under two different measures, $\mathbb{P}_{s}$ and $\mathbb{P}_{q}$.

Note that the statement of Lemma 4.1 old is actually correct in the sense that it is true, but it is very weak. In particular, in the proof of Theorem 2.6 in $\left[\mathrm{BDK}^{+} 18\right]$ Lemma 4.1 is required because Lemma 4.1 old is not sufficient.

Proof of Lemma 4.1. For the natural filtrations of the processes $X$ and $Y$ we have an inclusion

$$
\mathscr{F}_{n}^{X} \supset \mathscr{F}_{n}^{Y}, \quad n \in \mathbb{N},
$$

since $Y$ is a function of $X$. For $n \in \mathbb{N}$ and bounded Borel functions $h_{j}: \mathbb{R} \rightarrow \mathbb{R}, j=1,2, \ldots, n$,

$$
\begin{aligned}
\mathbb{E}_{S}\left[\prod_{j=1}^{n} h_{j}\left(Y_{k+j}\right) \mid \mathscr{F}_{k}^{X}\right]= & \mathbb{E}_{X_{k}} \prod_{j=1}^{n} h_{j}\left(f\left(X_{j}\right)\right) \\
= & \int_{S} Q\left(x_{0}, d x_{1}\right) h_{1}\left(f\left(x_{1}\right)\right) \int_{S} Q\left(x_{1}, d x_{2}\right) h_{2}\left(f\left(x_{2}\right)\right) \ldots \\
& \times\left.\int_{S} Q\left(x_{n-1}, d x_{n}\right) h_{n}\left(f\left(x_{n}\right)\right)\right|_{x_{0}=X_{k}}
\end{aligned}
$$

To transform the last integral, we introduce a new kernel: for $y \in f(S)$ chose $x \in S$ with $f(x)=y$, and then for $B \in \mathscr{B}(\mathbb{R})$ define

$$
\bar{Q}(y, B)=Q\left(x, f^{-1}(B)\right) .
$$

The expression on the right-hand side does not depend on the choice of $x$ because of (1). To make the kernel $\bar{Q}$ defined on $\mathbb{R} \times \mathscr{B}(\mathbb{R})$, we set

$$
\bar{Q}(y, B)=\mathbb{1}_{\{0 \in B\}}, y \notin f(S) .
$$

Then, setting $z_{n}=f\left(x_{n}\right)$, we obtain from the change of variables formula for the Lebesgue integral that

$$
\int_{S} Q\left(x_{n-1}, d x_{n}\right) h_{n}\left(f\left(x_{n}\right)\right)=\int_{\mathbb{R}} \bar{Q}\left(f\left(x_{n-1}\right), d z_{n}\right) h_{n}\left(z_{n}\right) .
$$

Likewise, setting $z_{n-1}=f\left(x_{n-1}\right)$, we get

$$
\begin{array}{rl}
\int_{S} & Q\left(x_{n-2}, d x_{n-1}\right) h_{n}\left(f\left(x_{n-1}\right)\right) \int_{S} Q\left(x_{n-1}, d x_{n}\right) h_{n}\left(f\left(x_{n}\right)\right) \\
= & \int_{S} Q\left(x_{n-2}, d x_{n-1}\right) h_{n}\left(f\left(x_{n-1}\right)\right) \int_{\mathbb{R}} \bar{Q}\left(f\left(x_{n-1}\right), d z_{n}\right) h_{n}\left(z_{n}\right) \\
= & \int_{\mathbb{R}} \bar{Q}\left(f\left(x_{n-2}\right), d z_{n-1}\right) h_{n}\left(z_{n-1}\right) \int_{\mathbb{R}} \bar{Q}\left(z_{n-1}, d z_{n}\right) h_{n}\left(z_{n}\right) .
\end{array}
$$

Proceeding further, we obtain

$$
\begin{gathered}
\int_{S} Q\left(x_{0}, d x_{1}\right) h_{1}\left(f\left(x_{1}\right)\right) \int_{S} Q\left(x_{1}, d x_{2}\right) h_{2}\left(f\left(x_{2}\right)\right) \ldots \int_{S} Q\left(x_{n-1}, d x_{n}\right) h_{n}\left(f\left(x_{n}\right)\right) \\
=\int_{\mathbb{R}} \bar{Q}\left(z_{0}, d z_{1}\right) h_{1}\left(z_{1}\right) \int_{\mathbb{R}} \bar{Q}\left(z_{1}, d z_{2}\right) h_{2}\left(z_{2}\right) \ldots \int_{\mathbb{R}} \bar{Q}\left(z_{n-1}, d z_{n}\right) h_{n}\left(z_{n}\right),
\end{gathered}
$$

where $z_{0}=f\left(x_{0}\right)$. 
Thus,

$\mathbb{E}_{S}\left[\prod_{j=1}^{n} h_{j}\left(Y_{k+j}\right) \mid \mathscr{F}_{k}^{X}\right]=\int_{\mathbb{R}} \bar{Q}\left(f\left(X_{k}\right), d z_{1}\right) h_{1}\left(z_{1}\right) \int_{\mathbb{R}} \bar{Q}\left(z_{1}, d z_{2}\right) h_{2}\left(z_{2}\right) \ldots \int_{\mathbb{R}} \bar{Q}\left(z_{n-1}, d z_{n}\right) h_{n}\left(z_{n}\right)$.

This equality and (2) imply that $Y$ is a Markov chain.

\section{Reference}

$\left[\mathrm{BDK}^{+} 18\right]$ Viktor B., Luca Di P., Tyll K., Mykola L., And Tomasz O. Asymptotic shape and the speed of propagation of continuous-time continuous-space birth processes. Adv. Appl. Probab., 50(1):74-101, 2018. 